\title{
El mercado laboral en bibliotecología y documentación: análisis de las ofertas de empleo publicadas en la lista de interés BIBGRA durante el periodo 2008-2014
}

\author{
Carlos Hugo Artaza \\ Universidad Nacional de Mar del Plata - UNMDP, Argentina.
}

ARTÍCULO

\begin{abstract}
Resumen
Con el objeto de analizar las características que presenta el mercado de trabajo en bibliotecología y documentación en la Argentina, se realizó un estudio de las ofertas laborales destinadas a bibliotecarios y licenciados en bibliotecología. Para ello se analizaron las propuestas de trabajo publicadas en la lista de interés BIBGRA durante el periodo 2008-2014. En la investigación se examinaron el número de empleos propuestos, la tipología de las organizaciones demandantes, los requisitos solicitados y la modalidad de los contratos. Se concluye que la mayor demanda de empleos proviene de organizaciones bibliotecarias. En su mayoría las organizaciones pertenecen al ámbito público. El tipo de contrato predominante es de carácter permanente. Con respecto a los conocimientos solicitados, las áreas de Tecnología de la Información, Organización y Tratamiento de la Información, Recursos y Servicios de Información e Idiomas figuran entre los más requeridos por los empleadores.
\end{abstract}

Palabras-clave

Mercado de trabajo ; Bibliotecología ; Documentación ; Bibliotecarios ; Documentalistas ; Licenciados ; Listas de interés ; BIBGRA ; Argentina

The labor market in librarianship and documentation: analysis of jobs advertised in the list of BIBGRA interest during the period 2008-2014

\begin{abstract}
The In order to determine the characteristics that have the job offers for graduates in Library and Information Science in Argentina were analyzed the jobs advertised in BIBGRA during the period 2008-2014. With offers were performed statistical analysis in relation to: number of jobs offered, typology of organizations applying, qualification requirements and type of contracts. We conclude that the largest number of job orders comes from library organizations. The applicants organizations are mostly of the public sector and the type of contract offer of a temporary nature. In relation to the knowledge required, the areas of Information Technology, Organization and Information Processing, Resources and Information Services and Language, are among the most demanded by employers.
\end{abstract}

Keywords

Labour market ; Library and information science ; Librarians ; Documentalists ; Interest lists ; BIBGRA ; Argentina

\section{Introducción}

En los últimos años, el mercado laboral del profesional en bibliotecología y documentación ha sido estudiado mediante metodologías y enfoques diversos. Especialistas de distintas nacionalidades se han ocupado de indagar las principales características que presentan las demandas laborales y también de analizar en detalle las competencias profesionales que adquieren los graduados durante su formación académica.

Entre los diversos autores que se han ocupado del tema podemos mencionar a Alonso y Vázquez (2000), Wainwright (2000), Breen y otros (2002), Chaín Navarro y otros (2002), Pearson y Urquhart (2002), Pineda (2002), Rocon Biancardi y otros (2002), Comalat, Espelt y Rubió (2003), Stilwell (2004), Abubakar (2005), Pando (2005), Azevedo y Gómez (2006), Choi y Rasmussen (2006), Kavulya (2007), Moreiro Gónzalez y otros 
(2009), Rodríguez Roche (2009), Espelt y Mañà (2011), Oliveira Lucas y Andrade Ouriques (2011) y Miranda Bressane y Vieira da Cunha (2011).

Estos investigadores, mediante diferentes metodologías de abordaje, han subrayado la importancia de identificar y describir debidamente los requerimientos (habilidades y conocimientos) que demanda la sociedad actualmente a los profesionales.

Los cambios tecnológicos, sociales y culturales desarrollados en las últimas décadas han generado una redefinición de los roles y funciones de los profesionales de la información.

Una sociedad que cambia requiere de una educación profesional en concordancia con las necesidades actuales. Es deseable que las competencias profesionales evolucionen y se adapten en armonía con las transformaciones del presente. Como sabemos, al finalizar su formación los graduados cuentan con determinadas competencias que se relacionan directamente con funciones propias de la profesión. Estas habilidades y conocimientos constituyen el principal recurso con que cuentan los egresados para enfrentar el reto que plantea la inserción laboral. En este contexto consideramos valioso analizar el mercado laboral.

Por tal motivo, en nuestra investigación nos propusimos indagar cuáles son las características principales que presentan las demandas de empleo destinadas a titulados en Bibliotecología y Documentación en Argentina a fin de evaluar las posibilidades de inserción laboral de los profesionales.

\section{Materiales y métodos}

Se utilizaron como fuente de datos para realizar el estudio, las ofertas de empleo publicadas en la lista de interés de la Asociación de Bibliotecarios de Buenos Aires (BIBGRA), uno de los principales foros electrónicos sobre bibliotecas y documentación de Argentina. El trabajo de carácter descriptivo, longitudinal y retrospectivo abarcó el periodo comprendido entre los años 2008 y 2014. Para recabar la información, se examinaron los archivos de e-mail disponibles en el sitio Web de la lista. Se analizaron las ofertas de empleo propuestas para bibliotecarios y licenciados en bibliotecología. Los ofrecimientos laborales destinados a estudiantes de las carreras no fueron incluidos en el estudio.

Con el objeto de comparar y analizar los requerimientos profesionales demandados, se relacionaron y procesaron estadísticamente los datos referidos a los siguientes ítems:

- Cantidad de ofertas laborales.

- Tipo de organización.

- Conocimientos requeridos

- Ámbito de organización (público o privado).

- Modalidad del contrato (temporario o permanente)

La clasificación de los conocimientos profesionales requeridos se efectuó principalmente a partir de las áreas temáticas recomendadas en las conclusiones del Tercer Encuentro de Directores y II de Docentes de Escuelas de bibliotecología y Ciencia de la Información del MERCOSUR (III Encuentro, 1998). Tambien se incluyeron los conocimientos pertenecientes a otras áreas profesionales como idiomas y estadística. En la tabla $n^{\circ} 1$ se presentan en detalle las Areas de Bibliotecología y Ciencias de la Información incluídas en el estudio: 


\begin{tabular}{|c|c|c|}
\hline \multicolumn{3}{|c|}{ Áreas de Bibliotecología y Ciencias de la Información } \\
\hline Área & Sigla & Descripción \\
\hline $\begin{array}{l}\text { Fundamentos teóricos de la } \\
\text { bibliotecología y la ciencia de } \\
\text { la información }\end{array}$ & $\mathrm{FBCl}$ & $\begin{array}{l}\text { Comunicación e información. Cultura y sociedad. Bibliotecología, } \\
\text { Documentación, Archivología, Museología, Ciencia de la Información } \\
\text { y áreas afines. Unidades y servicios de información. El profesional de } \\
\text { la información: formación y actuación. Historia y tendencias de la } \\
\text { producción de los registros del conocimiento, de las unidades y de los } \\
\text { sistemas nacionales e internacionales de información. }\end{array}$ \\
\hline $\begin{array}{l}\text { Procesamiento de la } \\
\text { información }\end{array}$ & OTI & $\begin{array}{l}\text { Organización del conocimiento y tratamiento de la información. } \\
\text { Tratamiento descriptivo de los documentos. Tratamiento temático: } \\
\text { teoría de la clasificación; análisis de la información; teoría de la } \\
\text { indización. Prácticas, tecnologías y productos. Generación y } \\
\text { organización de instrumentos de recuperación de la información. }\end{array}$ \\
\hline $\begin{array}{l}\text { Recursos y servicios de } \\
\text { información }\end{array}$ & RSI & $\begin{array}{l}\text { Fundamentos, principios, procesos e instrumentos para: selección, } \\
\text { adquisición, evaluación, descarte y relegamiento, preservación, } \\
\text { conservación y restauración de recursos de información } \\
\text { documentales y virtuales. Normativa relativa al desarrollo de las } \\
\text { colecciones. Fuentes de información documentales y virtuales: } \\
\text { conceptos, tipologías, características, acceso, utilización y evaluación. } \\
\text { Estudio y educación de usuarios. La industria de la información: } \\
\text { generación, producción y comercialización de documentos, fuentes y } \\
\text { servicios de información. Servicios de provisión y acceso. Servicios } \\
\text { de referencia e información. Servicios de extensión y acción cultural }\end{array}$ \\
\hline Tecnología de la información & $\mathrm{TI}$ & $\begin{array}{l}\text { Aplicaciones de la tecnología de la información y comunicación en las } \\
\text { unidades de información: análisis, evaluación y desarrollo (hardware y } \\
\text { software). Gestión de bases de datos y bibliotecas virtuales. Análisis y } \\
\text { evaluación de sistemas y redes de información. Informatización de las } \\
\text { unidades de información }\end{array}$ \\
\hline $\begin{array}{l}\text { Gestión de unidades de } \\
\text { información }\end{array}$ & GUI & $\begin{array}{l}\text { Teoría general de la administración: teoría organizacional, teoría de } \\
\text { sistemas. Técnicas modernas de gestión. Gestión de unidades y } \\
\text { servicios de información: lectores, usuarios, clientes y ambiente } \\
\text { social; formulación de proyectos de información; gestión de recursos } \\
\text { humanos; gestión financiera; gestión de espacio físico; mediación y } \\
\text { evaluación de servicios y unidades de información. }\end{array}$ \\
\hline Investigación & INV & $\begin{array}{l}\text { Epistemología de la investigación científica. Metodología de la } \\
\text { investigación social. Investigación en Bibliotecología y Ciencia de la } \\
\text { Información: producción y comunicación científica. }\end{array}$ \\
\hline
\end{tabular}

Tabla N N 1. Áreas de Bibliotecología y Ciencias de la Información. Fuente: Tercer Encuentro de Directores y II de Docentes de Escuelas de bibliotecología y Ciencia de la Información del MERCOSUR (III Encuentro,1998).

\section{Resultados}

\subsection{Cantidad de empleos ofertados}

Se registraron un total de 129 ofertas. La mayor proporción correspondió a pedidos de bibliotecarios (87,59\%) y la menor cantidad a licenciados en bibliotecología. (12,40\%). En la tabla $n^{\circ} 2$ se presenta el detalle de las solicitudes de empleo publicadas por cada año analizado:

\begin{tabular}{|c|c|c|c|c|c|}
\hline BIBGRA & \multicolumn{2}{|c|}{ Bibliotecario } & \multicolumn{2}{c|}{ Licenciado } & Totales \\
\hline ANO & Cantidad & Porcentaje & Cantidad & Porcentaje & 29 \\
\hline 2008 & 27 & 93,10 & 2 & 6,8 & 17 \\
\hline 2009 & 14 & 82,35 & 3 & 17,64 & 14 \\
\hline 2010 & 12 & 85,71 & 2 & 14,28 & 28 \\
\hline 2011 & 24 & 85,71 & 4 & 11,11 & 18 \\
\hline 2012 & 16 & 88,88 & 2 & 11,76 & 17 \\
\hline 2013 & 15 & 88,23 & 2 & 16,66 & 6 \\
\hline 2014 & 5 & 83,33 & 1 & $12,40 \%$ & 129 \\
\hline TOTAL & 113 & $87,59 \%$ & 16 & & \\
\hline
\end{tabular}




\subsection{Tipos de Organizaciones que realizan ofertas}

Las organizaciones que ofrecen puestos de trabajo presentan variantes en su tipología. En las ofertas para bibliotecarios, sobre un total de 113 ofrecimientos, el $91,15 \%$ correspondió a bibliotecas y un 4,42\% a centros de documentación y empresas. Con respecto a las ofertas de las bibliotecas, en su mayoría correspondieron a bibliotecas públicas (23\%), escolares primarias $(17,69 \%)$ y bibliotecas universitarias $(17,69 \%)$.

Las ofertas para licenciados presentan características similares. Sobre un total de 16 ofrecimientos el 93,75\% correspondió a bibliotecas y el 6,25 \% a centros de documentación. En relación a las bibliotecas, el mayor porcentaje correspondió a bibliotecas universitarias $(31,25 \%)$ y públicas $(12,5 \%)$. En el caso de los centros de documentación se registró una única oferta proveniente del ámbito de las ciencias económicas. En las siguientes tablas se presentan en detalles los diferentes tipos de organizaciones que ofrecen puestos de trabajo:

\begin{tabular}{|c|c|c|c|c|c|c|}
\hline BIBGRA & \multicolumn{2}{|c|}{ Bibliotecario } & \multicolumn{2}{|c|}{ Licenciado } & & \\
\hline Tipo de organización & Cantidad & Porcentaje & Cantidad & Porcentaje & Totales & Porcentaje \\
\hline Bibliotecas & 103 & 91,15 & 15 & 93,75 & 118 & 91,47 \\
\hline Centros de documentación & 5 & 4,42 & 1 & 6,25 & 6 & 4,65 \\
\hline Empresa & 5 & 4,42 & 0 & 0,00 & 5 & 3,87 \\
\hline Totales & 113 & $87,59 \%$ & 16 & $12,40 \%$ & 129 & $100 \%$ \\
\hline
\end{tabular}

Tabla № 3. Resumen de los Tipos de Organización que ofertan empleos.

\begin{tabular}{|l|c|c|c|c|c|c|}
\hline \multicolumn{1}{|c|}{ BIBGRA } & \multicolumn{2}{|c|}{ Bibliotecario } & \multicolumn{2}{c|}{ Licenciado } & \\
\hline \multicolumn{1}{|c|}{ Tipo de organización } & Cantidad & Porcentaje & Cantidad & Porcentaje & Totales & Porcentaje \\
\hline Biblioteca Nacional & 5 & 4,42 & 2 & 12,5 & 7 & 5,42 \\
\hline $\begin{array}{l}\text { Biblioteca Administración } \\
\text { Pública }\end{array}$ & 2 & 1,76 & 1 & 6,25 & 3 & 2,32 \\
\hline Biblioteca Medicina & 5 & 4,42 & 1 & 6,25 & 6 & 4,65 \\
\hline Biblioteca Escolar Primaria & 20 & 17,69 & 0 & 0,00 & 20 & 15,50 \\
\hline Biblioteca Escolar Secundaria & 10 & 8,84 & 0 & 0,00 & 10 & 7,75 \\
\hline Biblioteca Escolar Terciaria & 3 & 2,65 & 1 & 6,25 & 4 & 3,10 \\
\hline Biblioteca Odontologia & 2 & 1,76 & 0 & 0,00 & 2 & 1,55 \\
\hline Biblioteca Música & 2 & 1,76 & 0 & 0,00 & 2 & 1,55 \\
\hline Biblioteca Psicología & 3 & 2,65 & 0 & 0,00 & 3 & 3,10 \\
\hline Biblioteca Ciencia y Tecnología & 3 & 2,65 & 2 & 12,5 & 5 & 3,87 \\
\hline $\begin{array}{l}\text { Biblioteca Ciencias de la } \\
\text { Educación }\end{array}$ & 1 & 0,88 & 1 & 6,25 & 2 & 1,55 \\
\hline $\begin{array}{l}\text { Biblioteca Administración } \\
\text { Judicial }\end{array}$ & 1 & 0,88 & 0 & 0,00 & 1 & 0,77 \\
\hline Biblioteca Pública & 26 & 23,00 & 2 & 12,5 & 28 & 21,70 \\
\hline Biblioteca Universitaria & 20 & 17,69 & 5 & 31,25 & 25 & 19,37 \\
\hline $\begin{array}{l}\text { Centro de Documentación } \\
\text { Estudios Latinoamericanos }\end{array}$ & 1 & 0,88 & 0 & 0,00 & 1 & 0,77 \\
\hline $\begin{array}{l}\text { Centro de Documentación } \\
\text { Estudios Navales }\end{array}$ & 1 & 0,88 & 0 & 0,00 & 1 & 0,77 \\
\hline $\begin{array}{l}\text { Centro Documentación } \\
\text { Económicas }\end{array}$ & 3 & 2,65 & 1 & 6,25 & 4 & 3,10 \\
\hline Empresa Arquitectura & 1 & 0,88 & 0 & 0,00 & 1 & 0,77 \\
\hline Empresa Consultoria & 1 & 0,88 & 0 & 0,00 & 1 & 0,77 \\
\hline Empresa Editorial & 1 & 0,88 & 0 & 0,00 & 1 & 0,77 \\
\hline Empresa Informática & 1 & 0,88 & 0 & 0,00 & 1 & 0,77 \\
\hline Empresa Multimedios & 113 & $8,8,59 \%$ & 16 & $12,40 \%$ & 129 & $100 \%$ \\
\hline
\end{tabular}

Tabla № 4. Análisis detallado de los Tipos de Organización que ofertan empleos.

\subsection{Conocimientos requeridos}

Los conocimientos requeridos para cubrir los puestos de trabajo presentan variaciones. Para los cargos de bibliotecario, sobre un total de 258 condiciones solicitadas, el 44,34\% correspondió a conocimientos vinculados con el área de Tecnología de la Información (TI), el 28,29\% a Organización y Tratamiento de la Información (OTI), el 22,86 \% a Recursos y Servicios de Información (RSI), el 6,20\% a Idiomas (IDM), el 3,87 \% a Fundamentos de Bibliotecología y Ciencias de la Información (FBCl) y el 1,16\% a Estadística (EST). 
Los conocimientos más pedidos en el área de Tecnología de la Información ( $\mathrm{TI}$ ) se relacionan con conocimientos generales de informática $(06,58 \%)$, Microisis $(5,03 \%)$ bases de datos $(4,26 \%)$, Winisis $(02,71 \%)$ y programas de gestión automatizada de bibliotecas (01,93\%). En el área Organización y Tratamiento de la Información (OTI) los conocimientos más solicitados se relacionan con catalogación $(6,30 \%)$, clasificación $(06,30 \%)$ e indización (4,26\%). En el área Recursos y Servicios de Información (RSI), los conocimientos más solicitados fueron referencia $(7,75 \%)$ formación de usuarios $(5,42 \%)$ y búsqueda y recuperación de información $(4,26 \%)$. Con respecto al área de Idiomas (IDM) los conocimientos más pedidos fueron el inglés $(5,42 \%)$, seguido por francés $(00,77 \%)$.

En el caso de las ofertas para licenciados sobre un total de 25 condiciones requeridas, el $40 \%$ de los conocimientos demandados correspondió al área Recursos y Servicios de Información (RSI), el 20 \% a Organización y Tratamiento de la Información (OTI), el 24\% a Gestión de Unidades de Información (GUI), el 12\% a Fundamentos de Bibliotecología y Ciencias de la Información ( $\mathrm{FBCl}$ ) y el 4 \% a Tecnología de la Información $(\mathrm{TI})$.

Con respecto al área Recursos y Servicios de Información (RSI) los conocimientos solicitados se relacionan con referencia $(16 \%)$, formación de usuarios $(20 \%)$ y promoción de la lectura $(4 \%)$. Los conocimientos más requeridos en el área Organización y Tratamiento de la Información (OTI) fueron catalogación (8\%), clasificación (08\%) e indización (04\%). Con respecto a Gestión de Unidades de Información (GUI) los conocimientos más solicitados fueron planeamiento y gestión (24\%).

En el área Fundamentos de Bibliotecología y Ciencias de la Información (FBCl) los conocimientos mayormente requeridos fueron de archivística (12\%). Con respecto al área Tecnología de la Información (TI) los conocimientos más solicitados fueron sobre Winisis (4\%).

En las tablas siguientes se presentan en detalles los diferentes tipos de conocimientos requeridos para cubrir los puestos de trabajo publicados en la lista:

\begin{tabular}{|l|c|c|c|c|}
\hline \multicolumn{1}{|c|}{ BIBGRA } & \multicolumn{2}{c|}{ Bibliotecario } & \multicolumn{2}{c|}{ Licenciado } \\
\hline \multicolumn{1}{|c|}{ Requisitos / Areas de conocimiento } & Cantidad & Porcentaje & Cantidad & Porcentaje \\
\hline Tecnología de la información (TI) & 97 & 37,59 & 1 & 4 \\
\hline Organización y tratamiento de la información (OTI) & 73 & 28,29 & 5 & 20 \\
\hline Idiomas (IDM) & 16 & 6,20 & 0 & 0,00 \\
\hline Recursos y servicios de información (RSI) & 59 & 22,86 & 10 & 40 \\
\hline Gestión de unidades de información (GUI) & 0 & 0,00 & 6 & 24 \\
\hline $\begin{array}{l}\text { Fundamentos de bibliotecología y ciencias de la información } \\
\text { (FBCI) }\end{array}$ & 10 & 3,87 & 3 & 12 \\
\hline Estadistica (EST) & 3 & 1,16 & 0 & 0,00 \\
\hline \multicolumn{1}{|c|}{ Total } & $\mathbf{2 5 8}$ & $\mathbf{1 0 0}$ & $\mathbf{2 5}$ & $\mathbf{1 0 0}$ \\
\hline
\end{tabular}

Tabla № 5. Resumen de los conocimientos solicitados en las ofertas de empleos.

\begin{tabular}{|c|c|c|c|c|c|}
\hline BIBGRA & & \multicolumn{2}{|c|}{ Bibliotecario } & \multicolumn{2}{|c|}{ Licenciado } \\
\hline Requisitos & Área & Cantidad & Porcentaje & Cantidad & Porcentaje \\
\hline Estadistica & EST & 3 & 1,16 & 0 & 0,00 \\
\hline Planeamiento / gestión & GUI & 0 & 0,00 & 6 & 24 \\
\hline Archivistica & $\mathrm{FBCl}$ & 10 & 3,87 & 3 & 12 \\
\hline Inglés & IDM & 14 & 5,42 & 0 & 0,00 \\
\hline Francés & IDM & 2 & 0,77 & 0 & 0,00 \\
\hline Formación de usuarios & $\mathrm{RSI}$ & 14 & 5,42 & 5 & 20 \\
\hline Búsqueda y recuperación de información & $\mathrm{RSI}$ & 11 & 4,26 & 0 & 0,00 \\
\hline Referencia & $\mathrm{RSI}$ & 20 & 7,75 & 4 & 16 \\
\hline Desarrollo de colecciones & $\mathrm{RSI}$ & 4 & 1,55 & 0 & 0,00 \\
\hline Promoción de la lectura & RSI & 10 & 3,87 & 1 & 4 \\
\hline Catalogación & OTI & 16 & 6,20 & 2 & 8 \\
\hline Clasificación & OTI & 16 & 6,20 & 2 & 8 \\
\hline Indización & OTI & 11 & 4,26 & 1 & 4 \\
\hline Formato MARC 21 & OTI & 7 & 2,71 & 0 & 0,00 \\
\hline Reglas AA2 & OTI & 10 & 3,87 & 0 & 0,00 \\
\hline LC & OTI & 2 & 0,77 & 0 & 0,00 \\
\hline CDD & OTI & 1 & 0,38 & 0 & 0,00 \\
\hline $\mathrm{CDU}$ & OTI & 10 & 3,87 & 0 & 0,00 \\
\hline
\end{tabular}

Tabla № 6. Análisis detallado de los conocimientos solicitados en las ofertas de empleos. 


\begin{tabular}{|c|c|c|c|c|c|}
\hline BIBGRA & & \multicolumn{2}{|c|}{ Bibliotecario } & \multicolumn{2}{|c|}{ Licenciado } \\
\hline Requisitos & Área & Cantidad & Porcentaje & Cantidad & Porcentaje \\
\hline Winisis & $\mathrm{TI}$ & 7 & 2,71 & 0 & 0,00 \\
\hline D-space & $\mathrm{TI}$ & 6 & 2,32 & 1 & 4 \\
\hline Aguapey & $\mathrm{TI}$ & 6 & 2,32 & 0 & 0,00 \\
\hline Digitalización & TI & 7 & 2,71 & 0 & 0,00 \\
\hline Diseño web & $\mathrm{TI}$ & 5 & 1,93 & 0 & 0,00 \\
\hline Winisis & $\mathrm{TI}$ & 7 & 2,71 & 0 & 0,00 \\
\hline Programa automatizacion bibliotecas & $\mathrm{TI}$ & 5 & 1,93 & 0 & 0,00 \\
\hline Micros oft Office / Internet & $\mathrm{TI}$ & 17 & 6,58 & 0 & 0,00 \\
\hline Bases de datos & $\mathrm{TI}$ & 11 & 4,26 & 0 & 0,00 \\
\hline Libbiweb & TI & 2 & 0,77 & 0 & 0,00 \\
\hline Koha & $\mathrm{TI}$ & 3 & 1,16 & 0 & 0,00 \\
\hline Rredes sociales & $\mathrm{TI}$ & 4 & 1,55 & 0 & 0,00 \\
\hline Mcroisis & $\mathrm{TI}$ & 13 & 5,03 & 0 & 0,00 \\
\hline Ddigibepe & $\mathrm{TI}$ & 2 & 0,77 & 0 & 0,00 \\
\hline Alephino & $\mathrm{TI}$ & 1 & 0,38 & 0 & 0,00 \\
\hline Bera & $\mathrm{TI}$ & 1 & 0,38 & 0 & 0,00 \\
\hline Totales & & 258 & $100 \%$ & 25 & $100 \%$ \\
\hline
\end{tabular}

Tabla № 6. Análisis detallado de los conocimientos solicitados en las ofertas de empleos (Continuación).

\section{4 Ámbito de las organizaciones y modalidades de contratos}

Con respecto al tipo de organización (pública o privada) que ofrece empleos, los resultados presentan algunas diferencias. Para puesto de bibliotecario el $53,98 \%$ se origina en ámbito público y el $46,01 \%$ restante en el ámbito privado. Esta tendencia difiere levemente en las ofertas publicadas para licenciados en donde el $56,25 \%$ proviene del ámbito privado y el $43,75 \%$ de las ofertas se originan en el ámbito estatal. En la siguiente tabla se presentan los resultados obtenidos:

\begin{tabular}{|c|c|c|c|c|}
\hline BIBGRA 2008-2014 & \multicolumn{2}{|c|}{ Bibliotecario } & \multicolumn{2}{c|}{ Cicenciado } \\
\hline Tipo de ambito & Cantidad & Porcentaje & 9 & Porcentaje \\
\hline Privado & 52 & 46,01 & 7 & 56,25 \\
\hline Publico & 61 & 53,98 & 16 & 43,75 \\
\hline Totales & 113 & $100 \%$ & $100 \%$ \\
\hline
\end{tabular}

Tabla № 7. Ámbito de las organizaciones.

En relación a la modalidad de los contratos (temporario o permanente) también encontramos diferencias. En ofertas para bibliotecarios el $73,45 \%$ correspondió a contratos permanentes y el $25,54 \%$ restante a contratos temporarios. Con respecto a los trabajos para licenciados esta tendencia se incrementa. El $87,05 \%$ de las ofertas publicadas se relaciona con puestos de carácter permanente y solo el $12,05 \%$ de los trabajos publicados eran de carácter temporario. En la tabla $N^{\circ} 8$ se presentan los resultados obtenidos:

\begin{tabular}{|c|c|c|c|c|}
\hline BIBGRA 2008-2014 & \multicolumn{2}{|c|}{ Bibliotecario } & \multicolumn{2}{c|}{ Licenciado } \\
\hline Tipo de contrato & Cantidad & Porcentaje & Cantidad & 12,5 \\
\hline Temporario & 30 & 26,54 & 2 & 87,5 \\
\hline Permanente & 83 & 73,45 & 14 & $100 \%$ \\
\hline Totales & 113 & $100 \%$ & 16 & $\%$. \\
\hline
\end{tabular}

Tabla Nº 8. Modalidad de los contratos.

\section{Conclusiones}

A partir de la investigación hemos podido conocer algunas de las características que presentan las solicitudes de empleo profesional en la Argentina. A través de los resultados comprobamos que las organizaciones que solicitan personal son en su mayoría bibliotecas. En referencia al ámbito al que pertenecen estas organizaciones (público o privado), los resultados muestran una distribución desigual. En el caso de los puestos para bibliotecario, el mayor número de ofertas provienen del ámbito estatal, mientras que en las solicitudes para 
licenciados, las ofertas proceden mayormente del ámbito privado. En relación al tipo de contrato, tanto para bibliotecarios como para licenciados, las ofertas empleo son en su mayoría de carácter permanente. Con respecto a los conocimientos requeridos en los ofrecimientos se observa que en el caso de las ofertas para bibliotecario, las condiciones solicitadas se vinculan mayormente con las áreas de Tecnología de la Información (TI), Organización y Tratamiento de la Información (OTI), Recursos y Servicios de Información (RSI), Idiomas (IDM), Fundamentos de Bibliotecología y Ciencias de la Información (FBCl) y Estadística (EST). En las ofertas para licenciados los conocimientos demandados se relacionan con las áreas de Recursos y Servicios de Información (RSI), Organización y Tratamiento de la Información (OTI), Gestión de Unidades de Información (GUI), Fundamentos de Bibliotecología y Ciencias de la Información (FBCl) y Tecnología de la Información (TI).

Cabe destacar que los resultados obtenidos son coincidentes con investigaciones previas que hemos realizado (Artaza, 2011a y 2011b). En estos estudios, efectuados sobre las ofertas de empleo publicadas en las listas de interés ABDAM e IWETEL, también encontramos en relación a los conocimientos solicitados, una mayor demanda de requerimientos vinculados con las áreas de Tecnología de la Información, Organización y Tratamiento de la Información e Idiomas.

Finalmente queremos indicar algunas líneas de investigación que consideramos se encuentran relacionadas con el estudio elaborado. Una de ellas se vincula con el estudio de los perfiles profesionales, competencias y contenidos curriculares de las áreas presentes en los planes de estudio. Consideramos importante evaluar estos puntos a fin de constatar su correlación con las demandas del mercado de trabajo. El segundo aspecto de relaciona con los estudios de inserción laboral de los egresados de las carreras. En este sentido creemos necesario el desarrollo de estudios sistemáticos que indaguen cómo se insertan laboralmente los profesionales y qué puestos ocupan en las organizaciones. También creemos valioso evaluar la formación profesional de posgrado, analizando las ofertas de especializaciones, maestrías y doctorados, atendiendo principalmente a su integración con las carreras de grado y a su vinculación con el mundo del trabajo.

Consideramos que las líneas de investigación planteadas complementan adecuadamente el estudio efectuado y ofrecen la posibilidad de profundizar el conocimiento sobre la formación profesional y sus posibilidades de inserción laboral en la Argentina.

\section{Bibliografía}

Abubakar, T. (2005). Reviewing the library and information science curriculum for effective performance in nigerian library services. Focus on International \& Comparative Librarianship, 36 (1), 25-29.

Alonso Arévalo, J. y Vázquez Vázquez, M. (2000). Características del comportamiento del mercado de trabajo en Biblioteconomía, Archivística y Documentación: bienio 98/99. Anales de Documentación, 3, 9-24.

Artaza, C. (2011 a) El mercado laboral de los titulados en Bibliotecología y Documentación: análisis de las ofertas de empleo publicadas en Argentina. Revista Investigación Bibliotecológica, 25(54), 17-36.

Artaza, C. (2011 b) El mercado laboral de los titulados en Biblioteconomía y Documentación: análisis de las ofertas de empleo publicadas en IWETEL durante el periodo 2000-2007. Biblios. Revista de Bibliotecología y Ciencias de la Información. 44, 111.

Azevedo L. J., Gomez, S. (2006) O mercado de trabalho para os profissionais da informacao no contexto de empresas brasileiras das regiones geográficas norte, nordeste e centro-oeste, Informação \& Sociedade 16(1). Recuperado el 21 de enero de 2014 de: http://www.ies.ufpb.br/ojs2/index.php/ies/article/view/457/1508.

Breen, C., Farragher, A., McQuaid, M., Callanan, M., y Burke, M. A. (2002). New information management opportunities in a changing world. Library Review, 51 (3), 127-138.

Chaín Navarro, C., Garrido Lova, J. López Lucas, E. y Martínez Pellicer, A. (2002) Las prácticas curriculares y extracurriculares realizadas por los estudiantes de la facultad de documentación de la universidad de Murcia (1991-2001). Revista General de Información y Documentación, 12 (2), 323-353.

Choi, Y., y Rasmussen, E. (2006). What is needed to educate future digital librarians: A study of current practice and staffing patterns in academic and research libraries. D-Lib Magazine, 12(9), 2-4.

Comalat Navarra, M.; Espelt Busquets, C. y Rubió Rodón, A. (2003), Nuevos yacimientos laborales para diplomados: análisis de los convenios de cooperación educativa de la Facultad de Biblioteconomía y Documentación, (UB), 1998-2002, en Revista de Biblioteconomía i Documentació, 33, 71-78. 
Espelt, C. y Maña, T. (2011) El valor añadido de las prácticas externas para los estudiantes y las instituciones: La experiencia de la Facultad de Biblioteconomía y Documentación de Barcelona. EN: World Library and Information Congress: 77th IFLA General Conference and Assembly, San Juan, Puerto Rico. Recuperado el 21 de abril de 2014 de: http://conference.ifla.org/ifla77

Kavulya, J. M. (2007). Training of library and information science (LIS) professionals in kenya: A needs assessment. Library Review, 56 (3), 208-223.

Miranda Bressane, J.; Vieira da Cunha, M. (2011) La profesión de bibliotecólogo: competencias que exige un mercado en transformación. Revista Interamericana de Biblioteco ᄀlogía, 34 (3),329-333.

Moreiro González, J. A., Sánchez-Cuadrado, S., Morato, J. y Moreno, V. (2009) Desarrollo de una aplicación ontológica para evaluar el mercado de trabajo español en Biblioteconomía y Documentación. Revista Española de Documentación Científica, 32 (1), 81-98.

Oliveira L., E.; Andrade Ouriques A.; (2011) Formação e competências do bacharel em biblioteconomia da UDESC: análise seguin Classificação Brasileira de Ocupações. Inf. Inf., Londrina, 16 (3), 166 - 190.

Pando, D. A. (2005) Formacaäo e demanda professional en tratamento tematico da informacao no Brasil: uma análise comparativa de conteúdos programáticos universitários e de concursos públicos em biblioteconomia. Tesis de maestría no publicada, Campus de Marilia, Universidade Estadual Paulista. Marilla, Brasil.

Pearson, A. y Urquhart, C. (2002). Health informatics: Working across the professional boundaries. Library Review, 51(3), 200210.

Pineda J. M. (2002), Realidad laboral de los bibliotecarios y bibliotecólogos egresados de la Escuela de Bibliotecología dependiente de la Facultad de Filosofía, de la Universidad Nacional de Córdoba -Argentina. Revista Biblios, $12,1-23$. Recuperado el 11 de enero de 2015 de: http://dialnet.unirioja.es/servlet/fichero articulo? codigo=254958\&orden=0.

Rocon Biancardi, A. M., Facini, B., Sarmento Rosemberg, D. y Pereira Ricardo, S. (2002) O cenário do mercado de trabalho em biblioteconomia na percepção dos empresários capixabas1. Perspect. Cienc. inf., 7 (2), 167-178.

Rodríguez Roche, S. (2009) Las competencias profesionales en las ciencias de la información. ACIMED, 1, (20), 1-17.

Stilwell, C. (2004) Alumni perceptions of a post graduate Information and Library Science Education programme at the University of Natal, South Africa. South African Journal of Libraries and Information Science, 70 (1), $20-29$.

Wainwright, M . (2000) A school for scandal?. Library Association Record; 102 (10), 277 - 297.

\section{Datos del autor}

\section{Carlos Hugo Artaza}

Licenciado en Bibliotecologia y Documentación, Especialista y Magister en Docencia Universitaria por la Universidad Nacional de Mar del Plata. Doctorando en Educacion por la Universidad Autónoma de Barcelona. Docente del Departamento de Documentación, Facultad de Humanidades, Universidad Nacional de Mar del Plata.

cartaza@mdp.edu.ar

Recibido - Received: 2016-04-25

Aceptado - Accepted: 2016-06-21

\section{(cc) $\mathrm{EY}$}

This work is licensed under a Creative Commons Attribution 4.0

United States License.

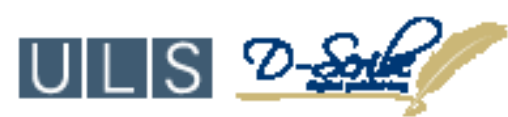

This journal is published by the University Library System of the University of Pittsburgh as part of its D-Scribe Digital Publishing Program and is cosponsored by the University of Pittsburgh Press. 